\title{
Prevalence of multimorbidity and associated treatment
}

\section{burden in primary care settings in Kerala: a cross-sectional}

\section{study in Malappuram District, Kerala, India [version 1; peer}

\section{review: 1 approved, 1 approved with reservations]}

\section{Sunaib Ismail (D), Antony Stanley (iD), Panniyammakal Jeemon (iD)}

Achutha Menon Centre for Health Science Studies, Sree Chitra Tirunal Institute for Medical Sciences and Technology, Trivandrum, Kerala, 695011, India

\author{
V1 First published: 23 Feb 2022, 7:67 \\ https://doi.org/10.12688/wellcomeopenres.17674.1 \\ Latest published: 04 May 2022, 7:67 \\ https://doi.org/10.12688/wellcomeopenres.17674.2
}

\section{Abstract}

Background: Multimorbidity or co-existence of two or more chronic conditions is common and associated with reduced quality of life and increased risk of death. We aimed to estimate the prevalence and pattern of multimorbidity in primary care settings in Kerala and the associated treatment burden, and quality of life.

Methods: A cross-sectional survey was conducted among 540 adult participants in Malappuram District, Kerala. A multi-stage cluster sampling method was employed. Hypertension, diabetes, chronic obstructive pulmonary disease, depression and anxiety screening were done by trained medical professionals. The remaining medical conditions were self-reported by the respondent and verified with patient held health records. The health-related quality of life [HRQoL] was measured using the EQ-5D-5L tool. The MTBQ tool was used for measuring the multimorbidity treatment burden. Logistic regression was used to identify variables associated with multi-morbidity. Results: Overall, the prevalence of multi-morbidity was 39.8\% (35.7 44.1). The prevalence of multi-morbidity among men (42.6\%) was relatively higher than that in women (38.1\%). Lower educational attainment, higher age group, and overweight or obesity status were independently associated with higher prevalence of multimorbidity. The most common pairs of coexisting chronic conditions reported in the study were hypertension and diabetes in males $(66.7 \%)$ and females $(70.8 \%)$. All domains of quality of life were impaired in individuals with multimorbidity.

Conclusion: Multimorbidity is a norm and affects two of five participants seeking care in primary care settings in Kerala. The social gradient in the prevalence of multimorbidity was evident with higher prevalence in individuals with low educational attainment. Multimorbidity seriously impairs quality of life and increases

\section{Open Peer Review Approval Status \\ 1 2 \\ version 2 \\ (revision) \\ 04 May 2022 \\ version 1 \\ 23 Feb 2022

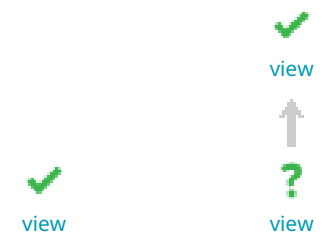 \\ 1. Soumya Gopakumar (ID), Government Medical College Thiruvananthapuram, Thiruvananthapuram, India \\ 2. Filipe Prazeres, University of Beira Interior, Covilhã, Portugal}

Any reports and responses or comments on the article can be found at the end of the article. 
treatment burden. The focus of management should move beyond individual diseases, and pivot towards interventions targeting multimorbidity management, with a specific focus for people living in lower socio-economic strata.

Keywords

Multimorbidity, Primary Care, Kerala, India

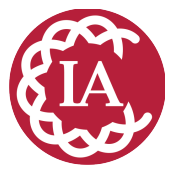

This article is included in the Wellcome

Trust/DBT India Alliance gateway.

\section{Corresponding author: Panniyammakal Jeemon (jeemon@sctimst.ac.in)}

Author roles: Ismail S: Conceptualization, Data Curation, Formal Analysis, Investigation, Methodology, Project Administration, Writing Original Draft Preparation, Writing - Review \& Editing; Stanley A: Data Curation, Formal Analysis, Writing - Original Draft Preparation, Writing - Review \& Editing; Jeemon P: Conceptualization, Funding Acquisition, Methodology, Resources, Supervision, Writing - Review \& Editing

Competing interests: No competing interests were disclosed.

Grant information: This work was supported by Wellcome through a Wellcome Trust/DBT India Alliance Clinical and Public Health Senior Fellowship [505229] to PJ. PJ also received research grants from the US National Heart Lung and Blood Institute for a work-sitebased cardiovascular risk reduction initiative (5R01 HL125442-05). PJ received research grants from the Australian National Health and Medical Research Council (1160283 and 1169766), the World Diabetes Federation (WDF-15-959), and the Indian Council of Medical Research for diabetes and hypertension risk reduction interventions. PJ also received research grants from Medical Research Council, UK for multi-morbidity research in Indian settings (MR/T037822/1).

The funders had no role in study design, data collection and analysis, decision to publish, or preparation of the manuscript.

Copyright: @ 2022 Ismail S et al. This is an open access article distributed under the terms of the Creative Commons Attribution License, which permits unrestricted use, distribution, and reproduction in any medium, provided the original work is properly cited.

How to cite this article: Ismail S, Stanley A and Jeemon P. Prevalence of multimorbidity and associated treatment burden in primary care settings in Kerala: a cross-sectional study in Malappuram District, Kerala, India [version 1; peer review: 1 approved, 1 approved with reservations] Wellcome Open Research 2022, 7:67 https://doi.org/10.12688/wellcomeopenres.17674.1

First published: 23 Feb 2022, 7:67 https://doi.org/10.12688/wellcomeopenres.17674.1 


\section{Introduction}

Multimorbidity is not uniformly defined in the health literature, but one of the straightforward definitions of the term is the co-existence of two or more chronic conditions in the same individual $^{1,2}$. Globally, knowledge on the epidemiology of multimorbidity is suboptimal since most of the available literature highlights comorbidity around a specific disease condition of interest or comorbid pairs in association with a single index disease $^{3}$. Further, available data are mostly focused on populations from high-income countries.

Globally, chronic diseases account for about 41 million deaths each year, equivalent to $71 \%$ of all deaths (World Health Organization, 2021). Out of these 41 million deaths, around 15 million occur prematurely in people aged 30-69 years. Notably, $85 \%$ of these premature deaths happen in low- and middle-income countries ${ }^{4}$. Death and disability attributable to chronic conditions are largely due to deteriorating health from the coexistence of more than two conditions. Despite the variations in the number of diseases' conditions included, and measures of multimorbidity across studies, findings suggest that approximately one in four adults have two or more chronic conditions, and half of older adults (60 years or above) have three or more chronic conditions ${ }^{1,5-8}$.

India does not have country-level estimates on multi-morbidity from its periodic national surveys. Marked variations in prevalence exist between available research studies on multimorbidity within the country. The overall prevalence of multimorbidity in adults was $28 \%$ in a study conducted in the state of Odisha 9 , while it was $58 \%$ among adults above the age of 50 years in a study conducted across 19 states in India 7 . In another study with data from seven states in India $^{10}$ the estimated prevalence of multimorbidity in older adults (>60 years) was $30 \%$.

In India, the epidemiological transition ratio, measured as the ratio of death and disability caused by communicable, maternal, neonatal, and nutritional diseases (CMNNDs) to those caused by non-communicable diseases (NCDs) and injuries, is lowest in the state of Kerala ${ }^{11}$. Consequently, we expect a relatively higher multimorbidity burden in Kerala as compared to the rest of the country. In a recent cross-sectional study conducted in one of the southern districts in Kerala, the estimated prevalence of multimorbidity at the community level was $45 \%{ }^{12}$.

The state government of Kerala recently introduced localized public health projects and government-sponsored pilots (Amrutham Arogyam) in selected districts after taking cognizance of the burden and the pattern of care-seeking in primary care settings (Arogya Keralam, 2022) The most recent primary care initiative is the ongoing phased transformation of primary health centres with high footfalls into family health centres with dedicated NCD clinics, mental health clinics, respiratory health clinics, stroke and hypertension management facilities ${ }^{13}$. However, an integrated approach to managing multimorbidity is yet to be introduced in the primary care settings of Kerala.

Estimating the burden of multimorbidity at the primary care level may further help in revamping the primary care delivery design to introduce integrated patient-centred care and reduce the associated treatment burden. We aimed to estimate the prevalence and pattern of multimorbidity in primary care settings in Kerala and study associated treatment burden, and quality of life.

\section{Methods}

\section{Ethics approval}

Ethics clearance for the study was obtained from the Institutional Ethics Committee of Sree Chitra Tirunal Institute for Medical Sciences and Technology (SCT/IEC/1587/NOVEMBER/2020). Participants were given an information sheet and consent form in the Malayalam language. Written informed consent was obtained from all participants before enrolling them in the study. The identity of the participants was kept anonymous. Participants with abnormal values requiring immediate care were notified to the respective Medical Officer in charge of the family health centre (FHC). All locally applicable protocols for COVID-19 prevention were strictly followed during the data collection.

\section{Study design}

The study followed a facility (family health centres of Malappuram district, Kerala) based cross-sectional design.

\section{Study setting}

We conducted the study in the FHCs of Malappuram district from 29.12.2020 to 09.02.2021. Malappuram is the most populated district in Kerala (total population $=4,112,920$ ) (Census, 2011) and is situated in the northern part of the state. Based on the National Family Health Survey - 5 (NFHS-5), the sex ratio of the district was 1101 . One in three women (66\%) in Malappuram district reported 10 or more years of schooling, which was comparatively lower than the state average of $77 \%$. The available studies on the prevalence of non-communicable diseases (NCD) indicate a high prevalence of comorbidities among individuals with diabetes in the district ${ }^{14}$. Administratively, the district is divided into 15 health blocks. At present, the integrated services of the non-communicable diseases control programme are implemented through the 59 fully functional FHCs and they are spread across 15 blocks of the district.

\section{Study population}

Adults (30-69) and older adults (70+) who were seeking care from the selected FHCs of Malappuram district were approached to be included in the study. The participants were residents of the district at least for the last twelve months. Those who did not give informed consent or were physically or mentally unfit to answer the questions and/or undergo clinical measurements or were pregnant/lactating mothers were excluded.

\section{Sample size}

Based on the previously available literature, we assumed a multi-morbidity prevalence of $28 \%$ in FHC settings ${ }^{15}$. The alpha and beta were kept at 0.05 and 0.20 for the sample size calculation. The sample size was calculated as 468 using the formula $\left((1.96)^{2} \mathrm{pq} / \mathrm{d}^{2}\right)^{*}$ design effect, where 'p' was the anticipated prevalence, ' $\mathrm{q}$ ' was ' $1-\mathrm{p}$ ' and ' $\mathrm{d}$ ' was the precision, which was taken as 0.05 . A design effect of 1.5 was applied as the sampling was multi-stage cluster sampling. We also accounted 
for a $10 \%$ non-response rate and estimated the final sample size as 515 .

\section{Sample selection}

There were 59 fully functional FHCs in the Malappuram district, spread across 15 health blocks. From these 15 blocks, 6 blocks were selected using computer-generated random numbers. Further, one FHC was randomly selected from each of the selected blocks using computer-generated random numbers. Subsequently, 15 participants who met the inclusion criteria were selected consecutively from each FHC during their outpatient visit, per day. This was continued for a week (6 days) in the same FHC (i.e., 90 participants per FHC).

\section{Data collection}

Data collection was done in the FHC setting. We used a structured interviewer-administered KoBo collect survey tool, which was prepared in English and then translated to Malayalam for data collection. Printed copies of the survey were placed in front of the participants for reference. It captured the sociodemographic factors, behavioural risk factors, and underlying medical conditions. Education was measured by capturing the highest level of formal education, and this variable was ordered into no formal schooling, primary education (Class I - Class VII), secondary education (Class VIII - Class X) and higher secondary or above.

The blood pressure (BP) was measured using a standard digital BP apparatus (Omron Blood pressure monitor-upper arm). Three readings were recorded at one-minute intervals in the nondominant arm. We measured the random blood sugar (RBS) by using a glucometer (Onetouch Verio Flex Meter) and capillary blood. Screening for depression, anxiety and chronic obstructive pulmonary disease (COPD) were done using a validated Malayalam version of the patient health questionnaire-9 (PHQ-9) ${ }^{16}$, generalized anxiety disorder-7 (GAD-7) ${ }^{17}$ assessment questionnaire and COPD population screener questionnaire ${ }^{18}$, respectively.

The remaining medical conditions viz. chronic kidney disease, cerebrovascular accident (CVA), coronary artery disease (CAD), heart failure, cataract, dementia, and cancer were self-reported by the respondent and verified with patient-held health records, provided by the patients. The patient held records were verified to confirm the comorbidity status. The patient held health records included consultation details (out-patient consultation form, discharge summaries), diagnostic details (specific test results), treatment details (drugs), and health insurance claims. For documenting treatment burden, we used a multi-morbidity treatment burden questionnaire (MTBQ) ${ }^{19}$. We assessed the health-related quality of life (HRQoL) by using the Malayalam version of the EQ-5D-5L instrument ${ }^{20}$ and derived the health status using the Indian 5-Level Version EQ-5D Value Set ${ }^{21}$.

Data collectors and quality control

A registered dental practitioner, who is undergoing post-graduate level training in public health, and two trained medical professionals were involved in data collection. The FHC medical officers and the staff nurses facilitated the data collection process.

\section{Operational definitions}

Multi-morbidity: The coexistence of two or more chronic conditions, from the listing of the following 12 conditions (hypertension, diabetes mellitus, depression, anxiety, COPD, chronic kidney disease, coronary artery disease (CAD), cerebrovascular accident (CVA), heart failure, cataract, dementia and cancer), in the same individual.

Hypertension: As per Joint National Committee-7 guidelines ${ }^{22}$.

Diabetes mellitus: A random capillary blood glucose value above $140 \mathrm{mg} / \mathrm{dl}$.

Depression: PHQ-9 score of 10 or above ${ }^{16}$.

Anxiety: GAD-7 score of 10 or above ${ }^{17}$.

Health-related quality of life (HRQoL): EQ-5D-5L ${ }^{20,21}$

Multimorbidity Treatment Burden: The MTBQ is a 10-item measure of treatment burden for patients with multimorbidity ${ }^{19}$. The study participants were categorized into high, medium, low or no treatment burden groups based on global MTBQ scores $(\geq 22=$ high, $10-22=$ medium, $<10=$ low or no treatment burden).

\section{Data management and data analysis}

The data collected via KOBO collect were downloaded and checked for incongruencies and missing data. Data cleaning was carried out using Microsoft Excel 2019. It was then imported to SPSS version 25 for analysis. Descriptive statistics were used to describe the study population by summarising the distribution of relevant variables. Continuous variables were presented as mean and standard deviation and categorical variables as proportions and percentages. One-way ANOVA was used to compare the differences in means of HRQoL and multimorbidity treatment burden across groups based on the number of chronic conditions. A backward stepwise logistic regression model was used to identify socio-demographic factors that were independently associated with multi-morbidity and generated odds ratio with its $95 \%$ confidence interval. The level of statistical significance was set at a p-value of less than 0.05 .

\section{Results}

Socio-demographic characteristics of the respondents

Overall, 540 participants completed the interview, out of which $324(60 \%)$ were women. No participants had missing data. The mean age of the study participants was 56.7 (12.0) years (Table 1). One in four participants reported no formal schooling $(26 \%)$, and more than half of the respondents were in the unemployed category $(52 \%)$. Three in five men $(64 \%)$ and women $(61 \%)$ were either overweight $(\mathrm{BMI}=23.0-24.9)$ or obese (BMI >25.0). Alcohol and tobacco use were reported only among men. 


\begin{tabular}{|c|c|c|c|}
\hline Variable & Total $(\mathrm{N}=540)$ & Men $(\mathrm{N}=\mathbf{2 1 6})$ & Women $(\mathrm{N}=324)$ \\
\hline Age (in years), mean (SD*) & $56.7(12.0)$ & $57.7(12.6)$ & $56.0(11.6)$ \\
\hline \multicolumn{4}{|l|}{ Age group, n (\%) } \\
\hline $30-49$ & $145(26.9 \%)$ & $57(26.4 \%)$ & $88(27.2 \%)$ \\
\hline $50-69$ & $308(57.0 \%)$ & $112(51.9 \%)$ & $196(60.5 \%)$ \\
\hline $70+$ & $87(16.1 \%)$ & $47(21.8 \%)$ & $40(12.3 \%)$ \\
\hline \multicolumn{4}{|l|}{ Educational status, n (\%) } \\
\hline No formal schooling & $139(25.7 \%)$ & $50(23.1 \%)$ & $89(27.5 \%)$ \\
\hline Primary (Class I - Class VII) & $231(42.8 \%)$ & $87(40.3 \%)$ & $144(44.4 \%)$ \\
\hline Secondary (Class VIII - Class X) & $123(22.8 \%)$ & $56(25.9 \%)$ & $67(20.7 \%)$ \\
\hline Higher secondary or above & $47(8.7 \%)$ & $23(10.6 \%)$ & $24(7.4 \%)$ \\
\hline \multicolumn{4}{|l|}{ Work Status, n (\%) } \\
\hline Government employee & $18(3.3 \%)$ & $7(3.2 \%)$ & $11(3.4 \%)$ \\
\hline Skilled Labourer & $27(5.0 \%)$ & $14(6.5 \%)$ & $13(4.0 \%)$ \\
\hline Unskilled/Manual Labourer & $118(21.9 \%)$ & $60(27.8 \%)$ & $58(17.9 \%)$ \\
\hline Homemaker & $95(17.6 \%)$ & $9(4.2 \%)$ & $86(26.5 \%)$ \\
\hline Unemployed & $282(52.2 \%)$ & $126(58.3 \%)$ & $156(48.1 \%)$ \\
\hline \multicolumn{4}{|l|}{ Tobacco and alcohol use, n (\%) } \\
\hline Smoking tobacco (ever use) & $57(10.6 \%)$ & $57(26.8 \%)$ & 0 \\
\hline Chewable tobacco (ever use) & $14(2.6 \%)$ & $14(6.4 \%)$ & 0 \\
\hline Any form of tobacco (ever use) & $71(13.1 \%)$ & $71(32.8 \%)$ & 0 \\
\hline Alcohol consumption (ever use) & $38(7 \%)$ & $38(17.5 \%)$ & 0 \\
\hline Body Mass Index, mean (SD) & $23.8(2.0)$ & $23.7(0.4)$ & $23.8(2.0)$ \\
\hline Overweight, n (\%) & $163(30.2 \%)$ & $67(31.0 \%)$ & $96(29.6 \%)$ \\
\hline Obese, n (\%) & $173(32.0 \%)$ & $71(32.9 \%)$ & $102(31.5 \%)$ \\
\hline
\end{tabular}

* SD $=$ Standard deviation

Prevalence and patterns of multi-morbidity

Prevalence of multi-morbidity. Among the study population, hypertension was the most prevalent morbidity $(68.1 \%)$ followed by diabetes (37.2\%) in both sexes (Table 2). One condition was reported by 229 (42.4\%) participants, while 165 (30.6\%) presented with two and $50(9.3 \%)$ participants reported three or more coexisting conditions. Overall, 215 participants (39.8\%) reported multi-morbidity (Figure 1). The prevalence of multimorbidity increased with age and decreased with an increase in the educational level of the study participants (Figure 2). Age-wise, the highest prevalence of multi-morbidity was in the 50-69 year age group. However, multi-morbidity was a notable problem even in the younger (30-49 years) population (19.3\%). The prevalence of multi-morbidity among men $(42.6 \%)$ was relatively higher than that in women $(38.1 \%)$.
In the multivariate analyses (Table 3), multi-morbidity was associated with the educational status, age group, and obesity. The propensity for multi-morbidity was higher in individuals in the higher age group, for 50-69 years (adjusted odds ratio, $\mathrm{aOR}=2.9 ; 95 \%$ CI: 1.5-5.4, $\mathrm{p}=0.01)$ and $70+$ years $(\mathrm{aOR}=3.0$; $95 \% \mathrm{CI}: 1.3-6.6, \mathrm{p}=0.01$ ) as compared to younger age groups. Similarly, propensity for multi-morbidity was higher in overweight/obese ( $\mathrm{aOR}=36.3$; 95\% CI: 20.7 - 63.7), $\mathrm{p}<0.001)$ individuals as compared to normal-weight individuals. Lower educational attainment was associated with multimorbidity. For example, the participants who had attained secondary education $(\mathrm{aOR}=0.43 ; 95 \% \mathrm{CI}: 0.21-0.89, \mathrm{p}=0.02)$ or higher secondary education or above $(\mathrm{aOR}=0.30 ; 95 \% \mathrm{CI}: 0.10-0.89, \mathrm{p}=0.03)$ had lower odds of multi-morbidity compared to individuals with no formal schooling. 
Table 2. Prevalence of individual conditions.

\begin{tabular}{|c|c|c|c|}
\hline Conditions & Total $(\mathrm{N}=540)$ & Men $(\mathrm{N}=\mathbf{2 1 6})$ & Women $(\mathrm{N}=324)$ \\
\hline Hypertension, n (\%) & $368(68.1 \%)$ & $146(67.5 \%)$ & $222(68.5 \%)$ \\
\hline Diabetes, n (\%) & $201(37.2 \%)$ & $82(37.9 \%)$ & $119(36.7 \%)$ \\
\hline COPD/Asthma, n (\%) & $32(5.9 \%)$ & $16(7.4 \%)$ & $16(4.9 \%)$ \\
\hline Renal diseases, n (\%) & $27(5.0 \%)$ & $12(5.5 \%)$ & $15(4.6 \%)$ \\
\hline$C V A^{*}, C A D^{*}$, or Heart failure & $27(5.0 \%)$ & $12(5.5 \%)$ & $15(4.6 \%)$ \\
\hline Cataract, n (\%) & $11(2.0 \%)$ & $6(2.8 \%)$ & $5(1.5 \%)$ \\
\hline Dementia, n (\%) & $7(1.3 \%)$ & $4(1.8 \%)$ & $3(0.9 \%)$ \\
\hline Cancer, n (\%) & $3(0.5 \%)$ & $1(0.5 \%)$ & $2(0.6 \%)$ \\
\hline Depression, n (\%) & $31(5.7 \%)$ & $9(4.2 \% \%)$ & $22(6.8 \%)$ \\
\hline Anxiety, n (\%) & $15(2.8 \%)$ & $6(2.8 \%)$ & $9(2.8 \%)$ \\
\hline \multicolumn{4}{|c|}{ * Depression was defined as a PHQ-9 score of 10 or above } \\
\hline \multicolumn{4}{|c|}{ \# Anxiety was defined as a GAD-7 score of 10 or above } \\
\hline - Cerebrovascular accident & y artery disease & & \\
\hline
\end{tabular}

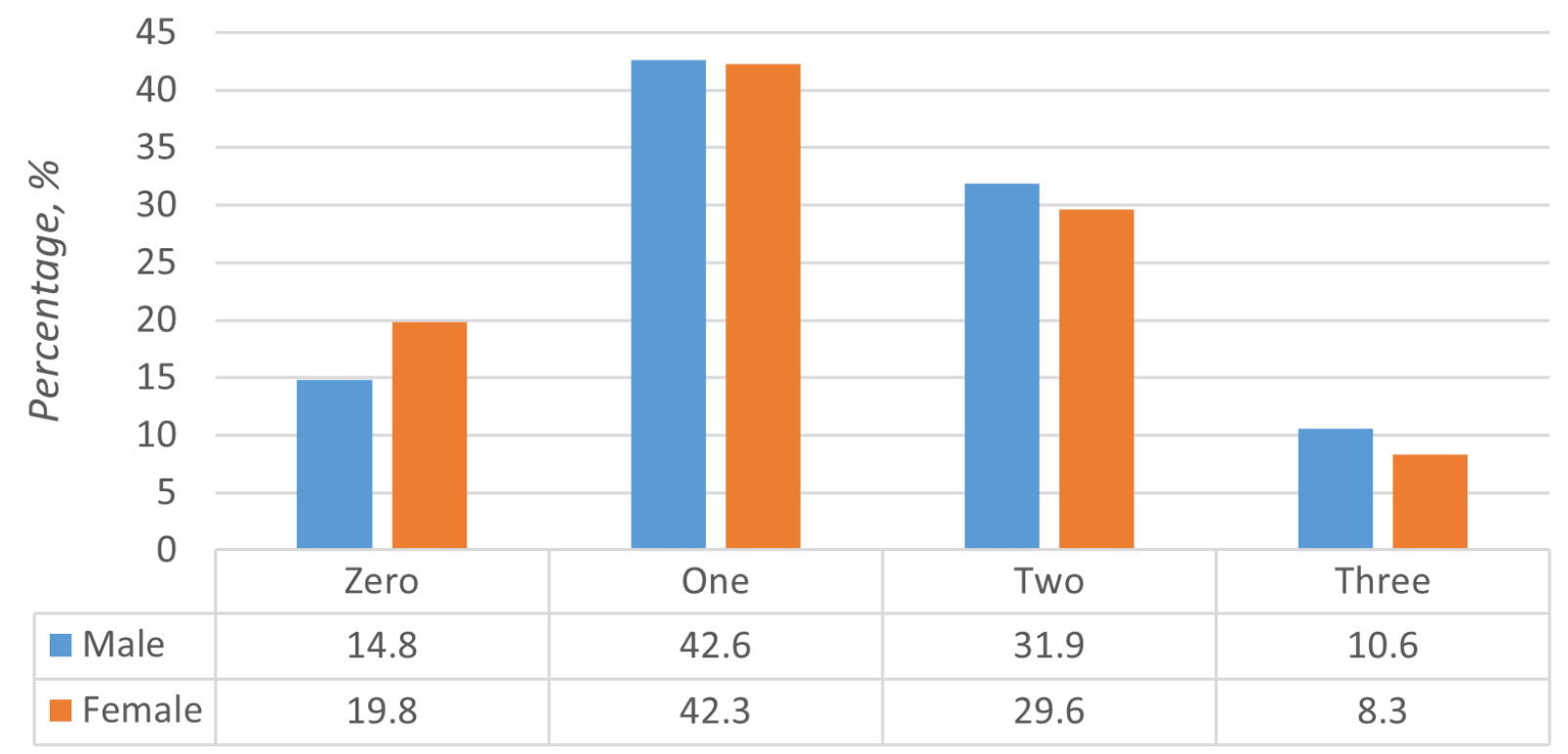

Number of conditions

Male Female

Figure 1. Multi-morbidity prevalence among males and females.

Patterns of multi-morbidity. The most common pairs of coexisting chronic conditions (Table 4) reported in the study were hypertension and diabetes in males $(66.7 \%)$ and females $(70.8 \%)$, followed by hypertension and COPD/asthma in males $(8.7 \%)$ and hypertension and depression in females (8.3\%). The most common triad in females was diabetes, hypertension, and 


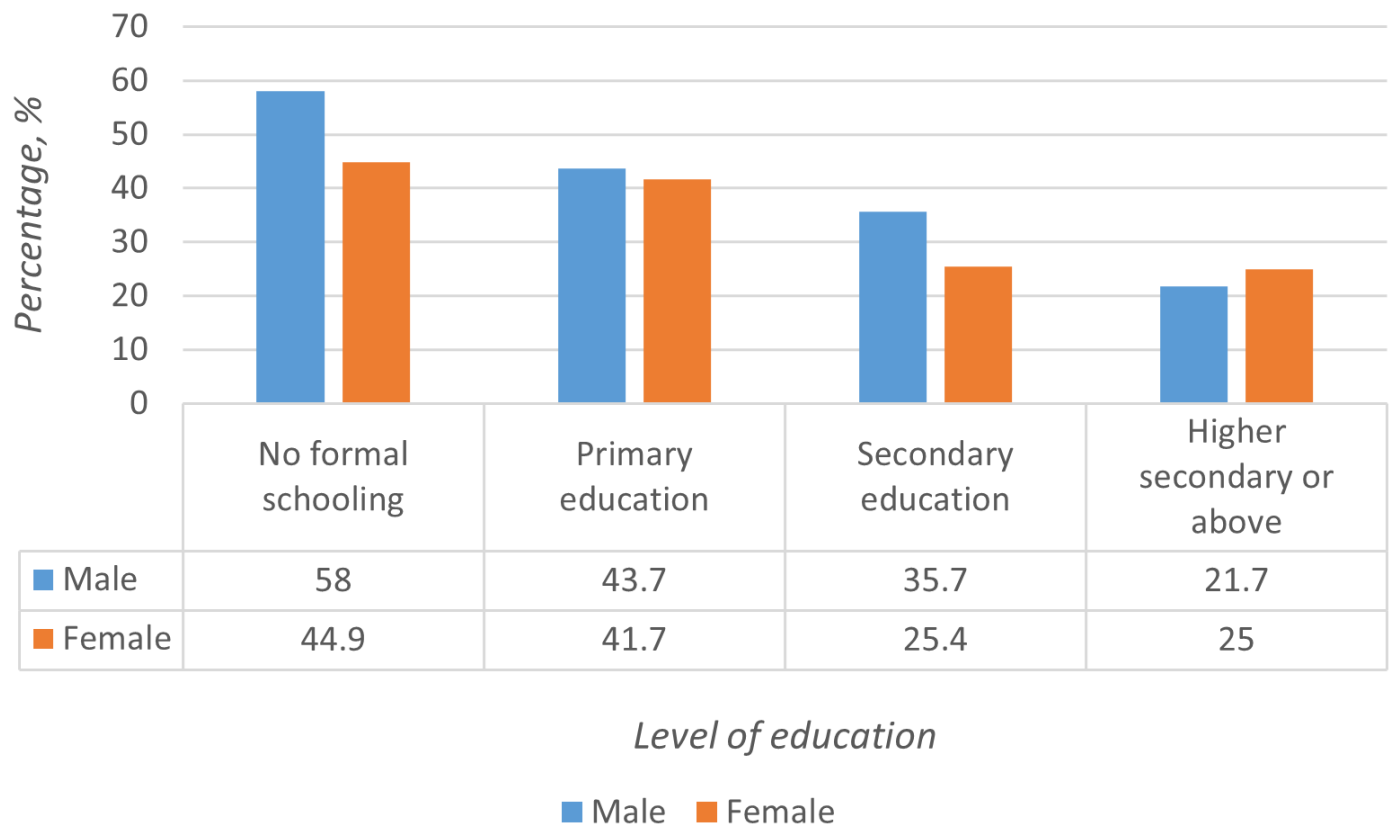

Figure 2. Prevalence of multi-morbidity based on education status.

Table 3. Association between socio-demographic characteristics and multimorbidity (MM).

\begin{tabular}{|c|c|c|c|c|}
\hline Variables & $\begin{array}{l}\text { MM present } \\
(\mathrm{N}=\mathbf{2 1 5})\end{array}$ & $\begin{array}{c}\text { MM absent } \\
(\mathrm{N}=325)\end{array}$ & Adjusted OR, 95\% CI & P value* \\
\hline \multicolumn{5}{|l|}{ Sex, n (\%) } \\
\hline Men & $92(42.6 \%)$ & $124(57.4 \%)$ & \multicolumn{2}{|l|}{ Reference group } \\
\hline Women & $123(38.1 \%)$ & $201(62.0 \%)$ & $0.71(0.44-1.17)$ & 0.28 \\
\hline \multicolumn{5}{|l|}{ Age group, n (\%) } \\
\hline 30-49 years & $28(19.4 \%)$ & $117(80.7 \%)$ & \multicolumn{2}{|l|}{ Reference group } \\
\hline 50-69 years & $146(47.4 \%)$ & $162(52.6 \%)$ & $2.87(1.52-5.42)$ & 0.001 \\
\hline $70+$ years & $41(47.1 \%)$ & $46(52.9 \%)$ & $2.97(1.32-6.63)$ & 0.008 \\
\hline \multicolumn{5}{|l|}{ Educational status, n (\%) } \\
\hline No formal schooling & $69(49.6 \%)$ & $70(50.4 \%)$ & \multicolumn{2}{|l|}{ Reference group } \\
\hline Primary (Class I - VII) & $98(42.4 \%)$ & $133(57.6 \%)$ & $0.80(0.45-1.44)$ & 0.46 \\
\hline Secondary (Class VIII - X) & $37(30.1 \%)$ & $86(69.9 \%)$ & $0.43(0.21-0.89)$ & 0.02 \\
\hline Higher secondary or above & $11(23.4 \%)$ & $36(76.6 \%)$ & $0.30(0.10-0.89)$ & 0.03 \\
\hline \multicolumn{5}{|l|}{ Obesity, n (\%) } \\
\hline Normal & $55(16.2 \%)$ & $284(83.8 \%)$ & \multicolumn{2}{|l|}{ Reference group } \\
\hline Overweight/ Obese & $160(79.6 \%)$ & $41(20.4 \%)$ & $36.27(20.67-63.65)$ & $<0.001$ \\
\hline
\end{tabular}


Table 4. Top three dyads and triads of multi-morbidity.

\begin{tabular}{|l|l|l|l|}
\hline Dyads, $\mathbf{n}(\mathbf{\%})$ & $\begin{array}{c}\text { Total } \\
(\mathbf{N = 1 6 5 )}\end{array}$ & $\begin{array}{c}\text { Men } \\
\mathbf{( N = 6 9 )}\end{array}$ & $\begin{array}{c}\text { Women } \\
\mathbf{( N = 9 6 )}\end{array}$ \\
\hline Hypertension - Diabetes & $114(69.1 \%)$ & $46(66.7 \%)$ & $68(70.8 \%)$ \\
\hline Hypertension - COPD/Asthma & $12(7.3 \%)$ & $6(8.7 \%)$ & $6(6.2 \%)$ \\
\hline Hypertension - Depression & $11(6.7 \%)$ & $3(4.3 \%)$ & $8(8.3 \%)$ \\
\hline Triads, n (\%) & $\begin{array}{c}\text { Total } \\
\mathbf{( N = 5 0 )}\end{array}$ & $\begin{array}{c}\text { Men } \\
(\mathbf{N}=\mathbf{2 3})\end{array}$ & $\begin{array}{c}\text { Women } \\
\mathbf{( N = 2 7 )}\end{array}$ \\
\hline Hypertension - Diabetes - COPD/Asthma & $7(18.9 \%)$ & $2(10.5 \%)$ & $5(27.8 \%)$ \\
\hline Hypertension - Diabetes - Heart disease & $7(18.9 \%)$ & $4(21.0 \%)$ & $3(16.7 \%)$ \\
\hline Hypertension - Anxiety - Depression & $6(16.2 \%)$ & $3(15.8 \%)$ & $3(16.7 \%)$ \\
\hline
\end{tabular}

\# The complete list of dyads and triads are attached as annexures in the supporting information section.

COPD/asthma (27.8\%), while in males it was diabetes, hypertension, and heart disease $(21.0 \%)$.

Association of multi-morbidity with quality of life and multi-morbidity treatment burden

Quality of life and multi-morbidity treatment burden. The HRQoL derived from the EQ-5D-5L deteriorated with an increase in the number of chronic conditions (Figure 3). Based on both HRQoL and the VAS score, individuals with multiple chronic conditions reported poor quality of life as compared to individuals with no or single chronic conditions (Table 5). All domains of quality of life such as mobility, usual care activities, depression/anxiety, self-care, pain/discomfort were impaired in individuals with multiple chronic conditions (Figure 3). The treatment burden associated with multi-morbidity showed a positive linear association with the number of co-existing chronic conditions (Table 5). Medium to high treatment burden was mostly noted in individuals with two and three or more chronic conditions (Figure 3) as compared to individuals with no or one chronic condition.

\section{Discussion}

We demonstrate that multimorbidity is almost a norm and prevalent in two in five participants seeking care from FHCs in Malappuram district, Kerala. The inverse relationship between educational status and multimorbidity, with the burden largely concentrated among the low education group, clearly highlights the reversal of social gradient in the prevalence of chronic disease conditions. Multimorbidity is a serious public health problem in the primary care settings in Kerala as it impairs the quality of life. The high treatment burden associated with multimorbidity could be partially attributable to the number of chronic conditions and partially to the lack of integrated care for the management of multimorbidity in the primary care system.

\section{Prevalence and patterns of multi-morbidity}

Lack of uniformity in the methods of data collection, study instruments, and the number of conditions covered across different studies limits the opportunity for direct comparison of the prevalence estimates with other studies. In a relatively comparable primary care-based study done in Odisha state, the prevalence of multi-morbidity was around $28 \%{ }^{23}$. The substantially higher prevalence of multimorbidity in our study is probably due to the advanced epidemiological transition state of Kerala as compared to Odisha ${ }^{11}$. It is now understood that areas in advanced epidemiological transition have a higher frequency of multi-morbidity ${ }^{11}$. Men reported a relatively higher prevalence of multi-morbidity than women among the set of morbidities studied, which contradicts previous research ${ }^{24,25}$. However, only men reported smoking and drinking in our study, which may have contributed to the increased frequency of multimorbidity among men. Further, exclusion of pregnant and lactating women from the study may have resulted in the underestimation of multimorbidity as conditions like depression is more common in antenatal and post-partum period ${ }^{26}$.

\section{Multi-morbidity status assessment with education status of the participants}

In recent studies from lower-middle-income countries, increasing levels of deprivation are consistently associated with a higher risk of multimorbidity ${ }^{27}$. In our study, the propensity for multi-morbidity was lower in the well-educated group as compared to individuals with poor educational attainment. Education is often considered a good proxy measure of a family's socioeconomic status ${ }^{28}$. Our findings highlight the importance of the social determinants of multimorbidity and call for integrated management programmes that incorporate strategies to address social determinants along with the medical needs. The vicious cycle of poor socio-economic status and high burden of NCDs is well established and it may further propel the burden of multimorbidity in the disadvantaged social groups.

\section{Multi-morbidity prevalence assessment in subgroups and their implications}

The age group of 50-69 years reported the highest prevalence of multimorbidity, which matched with findings from other studies $^{29}$. Even though five out of ten participants in the older 

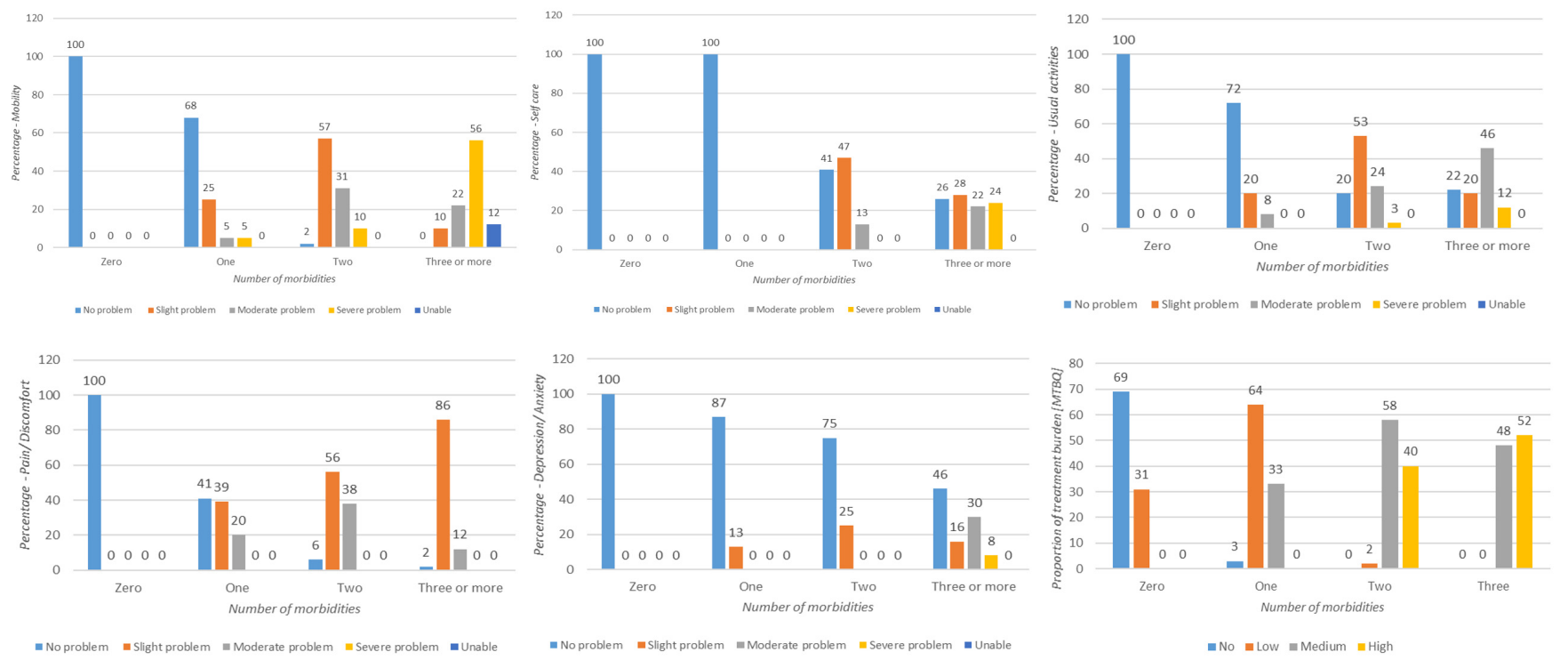

Figure 3. EQ-5D-5L domains, multimorbidity treatment burden and multimorbidity.

Table 5. Quality of life and treatment burden according to number of chronic conditions.

\begin{tabular}{|l|l|l|l|l|l|}
\hline \multirow{2}{*}{ Characteristic } & \multicolumn{3}{|c|}{ Number of multimorbid conditions } \\
\hline & $\begin{array}{l}\text { Zero } \\
(\mathbf{N = 9 6 )}\end{array}$ & $\begin{array}{l}\text { One } \\
(\mathbf{N = 2 2 9 )}\end{array}$ & $\begin{array}{l}\text { Two } \\
\mathbf{( N = 1 6 5 )}\end{array}$ & $\begin{array}{l}\text { Three or more } \\
\mathbf{( N = 5 0 )}\end{array}$ & P-Value* \\
\hline Quality of life, mean (SD) & $1(0.0)$ & $0.7(0.1)$ & $0.5(0.1)$ & $0.2(0.2)$ & $<0.001$ \\
\hline VAS scores, mean (SD) & $87.1(6.3)$ & $89.3(7.2)$ & $71.1(3.7)$ & $58.6(7.9)$ & $<0.001$ \\
\hline Multi-morbidity treatment burden, mean (SD) & $1.6(3.1)$ & $9.7(3.8)$ & $19.8(5.8)$ & $23.3(5.6)$ & $<0.001$ \\
\hline
\end{tabular}

* P-value calculated using One way ANOVA. SD: Standard deviation

age groups (50-69 years and 70/70+ years) reported multimorbidity, it was also prevalent in two of five in the younger age groups (30-49 years). Multimorbidity in the young adult age group may result in disproportionately high productivity loss as compared to other groups. Further, the progression of multimorbidity with more conditions later in life may adversely impact the health and productivity of these individuals. However, it is not clear how multimorbidity progresses in individuals and we need to generate data from well-designed cohort studies or registries of patients with multimorbidity on a regular follow-up to understand the progression. Integrated management with a focus on prevention may help to reduce the burden of multimorbidity in primary care settings. Novel models of prevention and control of multimorbidity need to be developed and evaluated before the wider adoption of these strategies in primary care settings. From the policy perspective, the identification of groups vulnerable to multimorbidity will help in the selection of preventive public health interventions to reduce the multimorbidity burden in high-risk groups.

\section{Multi-morbidity, quality of life and treatment burden and their implications}

Diabetes and hypertension were the most common coexisting chronic illnesses in both males and females in our study. This is consistent with the earlier multi-morbidity studies from India $^{30}$. Hypertension was the associated comorbidity in the most prevalent dyads and triads. In our study, multimorbidity in any combination impaired the quality of life and increased 
the treatment burden. A comparison with existing literature shows that similar findings are consistently seen across the world $^{31,32}$. It highlights that multimorbidity is a serious universal public health challenge and the lack of recognition of multimorbidity may potentially increase the disability associated with chronic conditions. New intervention models that could improve the quality of life of patients with multimorbidity need to be developed and evaluated in primary care settings.

\section{Strength and limitations}

The major strength of our study was the high response rate. In addition, we actively screened several chronic conditions, rather than relying on self-reported disease conditions. A medically qualified team conducted the screening, and the self-reported information was also cross-checked for accuracy. The research team explored the HRQoL using the EQ-5D-5L value set for India, which helps in overcoming the cultural and geographical biases associated with using value sets of other countries. The major limitation of the study was that the severity of chronic illnesses was not taken into consideration in the multi-morbidity assessment. Further, this is a cross-sectional study, and therefore the causality of the association cannot be established.

\section{Conclusion}

Multi-morbidity is a norm and affects two out of five participants seeking care in primary care settings in Kerala. Hypertension and diabetes are the most common co-existing conditions. The social gradient in the prevalence of multimorbidity was evident with higher prevalence in individuals with low educational attainment. Multimorbidity seriously impairs quality of life and increases treatment burden to the affected individuals. The focus of management should move beyond individual diseases, and pivot towards interventions targeting multimorbidity management, with a specific focus for people living in lower socio-economic strata and younger age groups to improve their quality of life.

\section{Data availability} Underlying data

figshare: Prevalence of multimorbidity, treatment burden and related quality of life in primary care settings in Kerala: A cross-sectional study in Malappuram, Kerala. https://doi. org/10.6084/m9.figshare. $17277167^{33}$
This project contains the following underlying data:

- Data_Multimorbidiy.xlsx (raw data)

figshare: Dataset for the study. https://doi.org/10.6084/ m9.figshare.17277167.v1 $1^{34}$

This project contains the following underlying data:

- Codes_Multimorbidiy (1).xlsx (data key)

Data are available under the terms of the Creative Commons Attribution 4.0 International license (CC-BY 4.0).

\section{Extended data}

figshare: Interview schedule in English with codes. https://doi. org/10.6084/m9.figshare.17274791.v1 ${ }^{35}$

figshare: Interview schedule in Malayalam with codes. https://doi. org/10.6084/m9.figshare.17274938.v1 ${ }^{36}$

figshare: Full list of dyads \& triads. https://doi.org/10.6084/ m9.figshare.17283761.v1 $1^{37}$

figshare: Consent form. https://doi.org/10.6084/ m9.figshare.18586052.v2 $2^{38}$

Data are available under the terms of the Creative Commons Attribution 4.0 International license (CC-BY 4.0).

\section{Author contributions}

Sunaib Ismail

Roles: Investigation, Methodology, Data collection, Data Curation, Formal Analysis, Writing - Original Draft Preparation

Antony Stanley

Roles: Data Curation, Formal Analysis, Co-writing Original Draft Preparation, Review and Editing

Panniyammakal Jeemon

Roles: Conceptualization, Funding Acquisition, Methodology, Resources, Supervision, Writing -Review \& Editing
1. Johnston MC, Crilly M, Black C, et al.: Defining and measuring multimorbidity: a systematic review of systematic reviews. Eur J Public Health. 2019; 29(1): 182-9.

PubMed Abstract | Publisher Full Text

2. MacMahon S, Calverley P, Chaturvedi N, et al.: Multimorbidity: a priority for global health research. The Academy of Medical Sciences: London, UK. 2018 127.

Reference Source

3. Boyd CM, Fortin M: Future of Multimorbidity Research: How Should
Understanding of Multimorbidity Inform Health System Design? Public Health Rev. 2010; 32(2): 451-74.

Publisher Full Text

4. Memon SA, Razak S, Weber I: Lifestyle Disease Surveillance Using Population Search Behavior: Feasibility Study. J Med Internet Res. 2020; 22(1): e13347.

PubMed Abstract | Publisher Full Text | Free Full Text

5. Nguyen H, Manolova G, Daskalopoulou C, et al.: Prevalence of multimorbidity in community settings: A systematic review and meta-analysis of 
observational studies. J Comorb. 2019; 9: 2235042X19870934. PubMed Abstract | Publisher Full Text | Free Full Text

6. Harrison C, Henderson J, Miller G, et al.: The prevalence of diagnosed chronic conditions and multimorbidity in Australia: A method for estimating population prevalence from general practice patient encounter data. PLOS One. 2017; 12(3): e0172935.

PubMed Abstract | Publisher Full Text | Free Full Text

7. Garin N, Koyanagi A, Chatterji S, et al.: Global Multimorbidity Patterns: A Cross-Sectional, Population-Based, Multi-Country Study.J Gerontol A Biol SC Med Sci. 2016; 71(2): 205-14.

PubMed Abstract | Publisher Full Text | Free Full Text

8. Abebe F, Schneider M, Asrat B, et al.: Multimorbidity of chronic noncommunicable diseases in low- and middle-income countries: A scoping review. J Comorb. 2020; 10: 2235042X20961919.

PubMed Abstract | Publisher Full Text | Free Full Text

9. Pati S, Swain S, Knottnerus JA, et al:: Magnitude and determinants of multimorbidity and health care utilization among patients attending public versus private primary care: a cross-sectional study from Odisha, India. Int J Equity Health. 2020; 19(1): 1-2.

PubMed Abstract | Publisher Full Text | Free Full Text

10. Mini GK, Thankappan KR: Pattern, correlates and implications of non communicable disease multimorbidity among older adults in selected Indian states: a cross-sectional study. BMJ Open. 2017; 7(3): e013529. PubMed Abstract | Publisher Full Text | Free Full Text

11. Dandona L, Dandona R, Kumar GA, et al.: Nations within a nation: variations in epidemiological transition across the states of India, 1990-2016 in the Global Burden of Disease Study. Lancet. 2017; 390(10111): 2437-60. PubMed Abstract | Publisher Full Text | Free Full Text

12. Jeemon CRP: Prevalence and patterns of multi-morbidity in the productive age group of 30-69 years: A cross-sectional study in Pathanamthitta District, Kerala. Wellcome Open Res. 2020; 5: 233.

PubMed Abstract | Publisher Full Text | Free Full Text

13. Krishnan GA, Nair AK: Primary health-care innovations with superior allusion to family health centers. Indian J Community Med. 2021; 46(1): 149-152.

PubMed Abstract | Publisher Full Text | Free Full Text

14. Zuhara NF, Banu AT, Chokli N: Gender wise prevalence of comorbidities and medication adherence among type 2 diabetics in Malappuram. IJPSR. 2019; 47(1): 4787-93.

15. Pati S, Swain S, Knottnerus JA, et al:: Health related quality of life in multimorbidity: a primary-care based study from Odisha, India. Health Qual Life Outcomes. 2019: 17(1): 116.

PubMed Abstract | Publisher Full Text | Free Full Text

16. Kroenke K, Spitzer RL, Williams JB: The PHQ-9: validity of a brief depression severity measure. J Gen Intern Med. 2001; 16(9): 606-13. PubMed Abstract | Publisher Full Text | Free Full Tex

17. Spitzer RL, Kroenke K, Williams JBW, et al.: A brief measure for assessing generalized anxiety disorder: the GAD-7. Arch Intern Med. 2006; 166(10): 1092-7.

PubMed Abstract | Publisher Full Text

18. Martinez FJ, Raczek AE, Seifer FD, et al.: Development and Initial Validation of a Self-Scored COPD Population Screener Questionnaire (COPD-PS). COPD. 2008; 5(2): 85-95.

PubMed Abstract | Publisher Full Text | Free Full Text

19. Duncan $P$, Murphy M, Man MS, et al.: Development and validation of the Multimorbidity Treatment Burden Questionnaire (MTBQ). BMJ Open. 2018; 8(4): e019413.

PubMed Abstract | Publisher Full Text | Free Full Text

20. Devlin N, Parkin D, Janssen B: Methods for analysing and reporting EQ-5D data [Internet]. Springer Nature; 2020. PubMed Abstract | Publisher Full Text
21. Jyani G, Sharma A, Prinja S, et al.: Development of an EQ-5D Value Set for India Using an Extended Design (DEVINE) Study: The Indian 5-Level Version EQ-5D Value Set. Value in Health. 2022. Publisher Full Text

22. Chobanian AV, Bakris GL, Black HR, et al:: Seventh Report of the Joint National Committee on Prevention, Detection, Evaluation, and Treatment of High Blood Pressure. Hypertension. 2003; 42(6): 1206-52. PubMed Abstract | Publisher Full Text

23. Pati S, Swain S, Metsemakers J, et al.: Pattern and severity of multimorbidity among patients attending primary care settings in Odisha, India. PLOS ONE. 2017; 12(9): e0183966.

PubMed Abstract | Publisher Full Text | Free Full Text

24. Abad-Díez JM, Calderón-Larrañaga A, Poncel-Falcó A, et al.: Age and gender differences in the prevalence and patterns of multimorbidity in the older population. BMC Geriatr. 2014; 14(1): 75

PubMed Abstract | Publisher Full Text | Free Full Text

25. Hussain MA, Huxley RR, Mamun AA: Multimorbidity prevalence and pattern in Indonesian adults: an exploratory study using national survey data. $B M$ J Open. 2015; 5(12): e009810.

PubMed Abstract | Publisher Full Text | Free Full Text

26. Bennett HA, Einarson A, Taddio A, et al:: Prevalence of Depression During Pregnancy: Systematic Review. Obstet Gynecol. 2004; 103(4): 698-709. PubMed Abstract | Publisher Full Text

27. Pathirana TI, Jackson CA: Socioeconomic status and multimorbidity: a systematic review and meta-analysis. Aust N Z J Public Health. 2018; 42(2) 186-94.

PubMed Abstract | Publisher Full Text

28. Broer M, Bai Y, Fonseca F: Socioeconomic inequality and educational outcomes: Evidence from twenty years of TIMSS. Springer Nature, 2019. Publisher Full Text

29. Pati S, Swain S, Hussain MA, et al:: Prevalence, Correlates, and Outcomes of Multimorbidity Among Patients Attending Primary Care in Odisha, India. Ann Fam Med. 2015; 13(5): 446-50.

PubMed Abstract | Publisher Full Text | Free Full Text

30. Singh K, Patel SA, Biswas S, et al.: Multimorbidity in South Asian adults: prevalence, risk factors and mortality. J Public Health (Oxf). 2019; 41(1): 80-9. PubMed Abstract | Publisher Full Text | Free Full Text

31. Wong ELY, Xu RH, Cheng AWL: Measuring the impact of chronic conditions and associated multimorbidity on health-related quality of life in the general population in Hong Kong SAR, China: A cross-sectional study, PLOS ONE. 2019; 14(11): e0224970.

PubMed Abstract | Publisher Full Text | Free Full Text

32. Williams JS, Egede LE: The Association Between Multimorbidity and Quality of Life, Health Status and Functional Disability. Am J Med Sci. 2016; 352(1): $45-52$.

PubMed Abstract | Publisher Full Text

33. Ismail S: Dataset for the study. figshare. Dataset. 2021 http://www.doi.org/10.6084/m9.figshare.17277167

34. Ismail S: Dataset for the study. figshare. Dataset. 2021 http://www.doi.org/10.6084/m9.figshare.17277167.v1

35. Ismail $\mathrm{S}$, Jeemon P, Stanley A: Interview schedule in English with codes. figshare. Dataset. 2021 http://wwww.doi.org/10.6084/m9.figshare.17274791.v1

36. Ismail S: Interview schedule in Malayalam with codes. figshare. Dataset. 2021.

http://www.doi.org/10.6084/m9.figshare.17274938.v

37. Ismail S: Full list of dyads \& triads. figshare. Dataset. 2021 http://www.doi.org/10.6084/m9.figshare.17283761.v1

38. Ismail S: Consent form. figshare. Dataset. 2022. http://www.doi.org/10.6084/m9.figshare.18586052.v2 


\section{Open Peer Review}

\section{Current Peer Review Status:}

Version 1

Reviewer Report 12 April 2022

https://doi.org/10.21956/wellcomeopenres.19552.r49832

(C) 2022 Prazeres F. This is an open access peer review report distributed under the terms of the Creative Commons Attribution License, which permits unrestricted use, distribution, and reproduction in any medium, provided the original work is properly cited.

\section{Filipe Prazeres}

Faculty of Health Sciences, University of Beira Interior, Covilhã, Portugal

Thank you for allowing me to read this interesting article regarding the prevalence of multimorbidity in India. As stated by the authors, most of the available data are mostly focused on populations from high-income countries, and so this data is of importance to add to the body of knowledge. Nonetheless, I have some comments to improve the manuscript:

- Since the year 2018 multimorbidity is a MeSH term ( https://www.ncbi.nlm.nih.gov/mesh/?term=multimorbidity). Please use multimorbidity Methods: instead of multi-morbidity throughout the paper.

Can the authors please explain why did they use the following criteria to diagnose Diabetes mellitus: A random capillary blood glucose value above $140 \mathrm{mg} / \mathrm{dl}$ ? The Criteria for the Screening and Diagnosis of Prediabetes and Diabetes ADA $2022^{1}$ when using Random plasma glucose is $\geq 200 \mathrm{mg} / \mathrm{dL}$ ( $11.1 \mathrm{mmol} / \mathrm{L}$ )

Why did the authors consider adults only the individuals >30years of age?

12 conditions were chosen by the authors to define multimorbidity. Can the authors please explain their choice criteria?

I also agree with Dr. Soumya Gopakumar that the authors need to clarify the definition of treatment burden. Sometimes the reader does not understand what the authors mean

Results when they use this term.

Figure 1 - The number of conditions stops at 3. There were no individuals with more than 3 co-occurring disorders?

Figure 3 is in a small format and because of that, it is difficult to read. 


\section{References}

1. American Diabetes Association: Standards of Medical Care in Diabetes-2022 Abridged for Primary Care Providers.Clin Diabetes. 2022; 40 (1): 10-38 PubMed Abstract | Publisher Full Text

Is the work clearly and accurately presented and does it cite the current literature? Yes

Is the study design appropriate and is the work technically sound? Partly

Are sufficient details of methods and analysis provided to allow replication by others? Partly

If applicable, is the statistical analysis and its interpretation appropriate? Yes

Are all the source data underlying the results available to ensure full reproducibility? Yes

Are the conclusions drawn adequately supported by the results? Yes

Competing Interests: No competing interests were disclosed.

Reviewer Expertise: multimorbidity; chronic diseases; family medicine; primary care; quality of life; geriatrics; public health

I confirm that I have read this submission and believe that I have an appropriate level of expertise to confirm that it is of an acceptable scientific standard, however I have significant reservations, as outlined above.

Author Response 22 Apr 2022

Panniyammakal Jeemon, Sree Chitra Tirunal Institute for Medical Sciences and Technology, Trivandrum, India

Thank you very much for the comments. We have changed 'multi-morbidity' to 'multimorbidity' throughout the manuscript.

We used the random glucose criteria to identify individuals with diabetes based on studies conducted in the Indian context (Susairaj P, Snehalatha C, Raghavan A, Nanditha A, Vinitha R, Satheesh K, Johnston DG, Wareham NJ, Ramachandran A. Cut-off Value of Random Blood Glucose among Asian Indians for Preliminary Screening of Persons with Prediabetes and Undetected Type 2 Diabetes Defined by the Glycosylated Haemoglobin Criteria. J Diabetes Clin Res. 2019;1(2):53-58, and Somannavar S, Ganesan A, Deepa M, Datta M, Mohan V. Random capillary blood glucose cut points for diabetes and pre-diabetes derived from community-based 
opportunistic screening in India. Diabetes Care. 2009 Apr;32(4):641-3.).

We included adults $>30 y e a r s$ of age in our study based on the Revised operational guidelines of the National Program for Prevention and Control of Cancer, Diabetes, CVD and Stroke ((Operational Guidelines of NPCDCS (Revised - 2013-17)_1.pdf (mohfw.gov.in)).

The twelve conditions that we have included in our study for multimorbidity assessment are selected from the Academy of Medical Sciences (UK) report on multimorbidity.

The treatment burden is assessed based on the Multimorbidity Treatment Burden Questionnaire (MTBQ). It is a validated 10-item questionnaire, which covers multiple domains of treatment burden such as coordinating healthcare appointments, taking multiple medicines, self-monitoring their health and making lifestyle changes.

The last category in Fig 1 was three or more conditions and we have corrected the error in the revised version. We have divided the Fig 3 into Fig 3A-3F and provided them separately.

Competing Interests: No competing interests were disclosed.

Reviewer Report 14 March 2022

https://doi.org/10.21956/wellcomeopenres.19552.r49022

(C) 2022 Gopakumar S. This is an open access peer review report distributed under the terms of the Creative Commons Attribution License, which permits unrestricted use, distribution, and reproduction in any medium, provided the original work is properly cited.

\section{Soumya Gopakumar}

Department of Community Medicine, Government Medical College Thiruvananthapuram, Thiruvananthapuram, Kerala, India

This is a well written article with suitable design. The authors have beautifully described the screening procedures used in the study which can be replicated. However I would like to point out that, the study was done in a single district in Kerala. The study subjects included patients attending family health centres only. In a state like Kerala, people depend on private health care providers also. In the present study multi-morbidity was lower in the well-educated group. Education alone may not be good proxy measure of a family's socioeconomic status especially in a district like Malappuram as many family members may be living abroad and adds to the family income. They may seek health in a private hospital. In sample size calculation, precision to be specified as $5 \%$ absolute precision. There is a lack of clarity in the term treatment burden.

Is the work clearly and accurately presented and does it cite the current literature? Yes

Is the study design appropriate and is the work technically sound? 
Yes

Are sufficient details of methods and analysis provided to allow replication by others? Yes

If applicable, is the statistical analysis and its interpretation appropriate?

Yes

Are all the source data underlying the results available to ensure full reproducibility?

Yes

Are the conclusions drawn adequately supported by the results?

Partly

Competing Interests: No competing interests were disclosed.

Reviewer Expertise: Public health, research methods

I confirm that I have read this submission and believe that I have an appropriate level of expertise to confirm that it is of an acceptable scientific standard.

Author Response 22 Apr 2022

Panniyammakal Jeemon, Sree Chitra Tirunal Institute for Medical Sciences and Technology, Trivandrum, India

Thank you very much for the positive comments. We have included the name of the district in the study title itself. We agree that we have not considered patients seeking primary care from private facilities in Kerala. We accept it as a limitation of the study. Educational level has been widely accepted as a proxy for socio-economic status (Reddy KS, Prabhakaran D, Jeemon P, Thankappan KR, Joshi P, Chaturvedi V, Ramakrishnan L, Ahmed F. Educational status and cardiovascular risk profile in Indians. Proc Natl Acad Sci U S A. 2007 Oct 9;104(41):16263-8.). It is also difficult to assess the income due to reporting errors. The treatment burden is estimated using a validated questionnaire, which assesses multiple domains of treatment burden such as coordinating healthcare appointments, taking multiple medicines, self-monitoring health and making lifestyle changes ( https://www.bristol.ac.uk/primaryhealthcare/resources/mtbq/).

Competing Interests: No competing interests were disclosed. 